\title{
Genome-Wide DNA Copy Number
}

National Cancer Institute

\section{Source}

National Cancer Institute. Genome-Wide DNA Copy Number. NCI Thesaurus. Code C104674.

The number of DNA sequence copies found over the entire genome of an individual. 